\title{
From phenotyping to the study of clonal relationship of microbial isolates
}

Carla Fontana ${ }^{1,2}$, Stefania Stefani ${ }^{3}$,Angelo Pan ${ }^{4,5}$, Barbara Pieretti $^{6}$, Gian Maria Rossolini ${ }^{7,8}$, Pietro Emanuele Varaldo', Mario Sarti ${ }^{10}$

Comitato di Studio per le Infezioni correlate all'Assistenza Sanitaria dell'AMCLI*

I Dipartimento di Medicina Sperimentale e Chirurgia, Università deglisStudi di Rome Tor Vergata - Roma

2 Microbiologia e Virologia, Fondazione PoliclinicoTor Vergata, Roma

3 Dipartimernto Di Scienze Bio-Mediche, Università degli Studi di Catania, Catania

4 Area Rischio Infettivo, Agenzia Sanitaria e Sociale Regionale dell'Emilia-Romagna, Bologna

5 Divisione di Malattie Infettive e Tropicali, Istituti Ospitalieri di Cremona

6 Laboratorio di Patologia Clinica, Ospedale "S. Croce", Azienda Ospedaliera Ospedali Riuniti Marche Nord, Fano

7 Dipartimento di Biotecnologie Mediche, Università di Siena

8 Dipartimento di Medicina Sperimentale e Clinica, Sezione di Medicina Critica e Medicine Specialistiche, Università di Firenze S.O.D. Microbiologia e Virologia, Azienda Ospedaliera Universitaria Careggi, Firenze

9 Sezione di Microbiologia, Dipartimento di Scienze Biomediche e Sanità Pubblica Università Politecnica delle Marche, Ancona

10 Dipartimento Interaziendale ad Attività Integrata Medicina di Laboratorio ed Anatomia Patologica, Microbiologia Clinica Provinciale Azienda USL di Modena NOCSAE, Baggiovara, Modena.

*Comitato di Studio per le Infezioni correlate all'Assistenza Sanitaria CoSIAS: Mario Sarti, Simone Ambretti, Giancarlo Basaglia, Susanna Cuccurullo, Carla Fontana, Lucina Fossati, Barbara Pierettti, Carlo Tascini

Key words: Outbreak, Molecular typing, Genotyping, Fingerprinting, Sequencing

\section{Dalla tipizzazione fenotipica allo studio della relazione clonale degli isolati microbici}

\section{SUMMARY}

The term "typing" is generally used with two meanings: a methods to establish the correct taxonomic collocation of a genus/specie/biotype, b methods for discriminating different bacterial isolates of the same species in order to establish the genetic relationship among the microorganisms involved in a possible outbreak. In this paper we focus our attention on the second aspect, that represents a relevant epidemiological tools in infection prevention and control.Typing systems are traditionally based on two steps workup: the first is the study of phenotypes such as serotype, biotype, phage-type, or antimicrobial susceptibilities of the isolates and this can be easily performed in every microbiology laboratory; the second, examines the relatedness of isolates at a molecular level. Over the years many molecular methods have been developed and efficiently applied in several hospital settings. The large panorama of methods put the microbiologists in trouble to operate the proper choice.

Thus, in the present paper, we have reviewed old as well new molecular typing methods in order to provide a useful guide that can represent an overview on molecular methods and particularly of their specific pro and cons.

\section{INTRODUZIONE}

Per tipizzazione dei microrganismi epidemiologia molecolare, si intende l'insieme delle procedure che si pongono l'obiettivo di stabilire l'identità o la correlazione clonale di ceppi microbici isolati da pazienti e/o substrati ad esempio nell'ambiente ospedaliero: letti, superfici, carrelli, strumentazioni, ecc... nel corso del tempo e che si sospetta possano essere coinvolti in focolai epidemici. Questo studio, oltre a conoscenze approfondite in ambito microbiologico, richiede un approccio multidisciplinare, che per essere efficace ai fini della prevenzione e/o delle misure d'intervento, deve poter vedere coinvolte tutte le figure cliniche ed ovviamente le direzioni sanitarie.

È opportuno precisare anzitutto alcune definizioni:

- Outbreak infettivo o fenomeno epidemico espressione anglosassone che significa "evento epidemico" definito come "un aumento statisticamente significativo della frequenza di infezione rispetto alla frequenza di infezione osservata precedentemente" $(7,1)$.

Indica dunque un numero di casi superiore all'at-

\section{Corresponding author: Carla Fontana}

Clinical Microbiology Laboratories; Polyclinic Tor Vergata, Rome V.le Oxford 8I, 00 I 33 Rome, Italy

Tel.: 039-6-20902I58 - Fax: 039-6-20902I59

E-mail: carla.fontana@uniroma2.it 
teso di una data infezione in un determinato periodo di tempo e all'interno di un determinato contesto o area geografica. Occorre precisare che anche un solo caso di un'infezione rara può costituire un outbreak. Nell'accezione comune questo termine viene ormai diffusamente utilizzato quando la numerosità dei casi coinvolti nell'evento epidemico e la loro estensione geografica è limitata riservando l'espressione epidemia alle situazioni in cui siano coinvolte aree piuttosto ampie o gruppi di popolazione numerosi.

- Cluster epidemico - con tale espressione si intende un aumento, statisticamente non significativo, di casi di infezione che condividono uno o più fattori di rischio (39).

- Caso - per definire un caso di infezione si devono considerare, all'interno dell'unità di tempo e luogo, specifici criteri clinico-microbiologici di inclusione o esclusione. A volte risulta necessario ricorrere ad una gradazione della sua definizione: caso certo, probabile caso o possibile caso. In alcune situazioni conviene inoltre distinguere tra casi di infezione e casi di colonizzazione (28).

- Clone - cellula o gruppo di cellule o organismo discendente da un singolo progenitore ancestrale rispetto a cui risulta geneticamente identico, come è il caso di una colonia batterica in cui tutte le cellule originano dalla replicazione di una singola cellula.

- Fingerprinting - "impronta" caratteristica del genoma di un organismo ottenuta mediante l'utilizzo di specifici marcatori.

Il termine tipizzazione, inteso come riconoscimento della clonalità degli isolati microbici, viene spesso usato in contrapposizione al concetto di tipizzazione tassonomica, pur esprimendo in realtà concetto ben diverso. Lo scopo degli studi di tassonomia è infatti quello di collocare un microrganismo in un raggruppamento taxon inteso a livello di famiglia, genere, specie o subspecie. In alcuni casi i metodi che consentono la classificazione tassonomica e quelli destinati alla caratterizzazione clonale possono essere gli stessi, ma vengono comunque usati con finalità profondamente diverse: classificare significare definire tutti i tratti di uguaglianza all'interno di un carattere, tipizzare significa valutare le divergenze all'interno dello stesso. Con il termine di tipizzazione si intende all'atto pratico una procedura basata sull'utilizzo di una o più metodiche atta ad accertare il grado di correlazione genetica di due $o$ più isolati.

Se i ceppi non risultano fra di loro geneticamente correlati il livello di correlazione è diverso a seconda del metodo utilizzato è improbabile che appartengano allo stesso fenomeno epidemico. È d'altronde praticamente impossibile stabilire se più pazienti siano coinvolti in un sospetto outbreak senza una attenta analisi epidemiologica, quindi indagine epidemiologica e tipizzazione clonale sono due diversi aspetti dello stesso problema.

Un metodo di tipizzazione deve possedere i seguenti requisiti:

1 applicabilità, intesa come la possibilità di tipizzare, con quella metodica, una determinata tipologia di microrganismi;

2 potere discriminante, ossia la capacità del metodo di discriminare i diversi genotipi nell'ambito di una specie;

3 riproducibilità e comparabilità intra e inter laboratorio, ossia la capacità del metodo di fornire lo stesso risultato di tipizzazione se ripetuto più volte o se effettuato in diversi laboratori;

4 di facile attuazione e che provveda informazioni di uso pratico;

5 flessibilità al fine di essere usato per diverse specie microbiche;

In ogni caso una metodica, per poter essere realisticamente utilizzabile nella pratica, deve risultare di eseguibilità sufficientemente semplice e rapida oltre che relativamente poco costosa.

Sostanzialmente i metodi di tipizzazione si distinguono in: fenotipici e genotipici.

\section{I metodi di tipizzazione fenotipica}

La tipizzazione fenotipica consiste nel determinare le caratteristiche comuni e quelle discriminanti espresse da due o più isolati appartenenti alla stessa specie. Questi metodi possono basarsi sulla caratterizzazione e sul confronto della struttura antigenica serotyping degli isolati, sulle loro proprietà fisiologiche e metaboliche biotyping, sulla sensibilità agli antimicrobici resistotyping, o sulla sensibilità alla lisi verso batteriofagi phagetyping. Del tutto recentemente si è aggiunta a queste metodiche più consolidate anche la possibilità di confrontare gli spettri proteici degli isolati mediante una tecnologia di recente acquisizione nel mondo microbiologico: la Matrix Assisted Laser Desorption/Ionization Time-of-Flight Mass Spectrometry MALDI-TOF-MS $(9,15,25)$.

In genere i metodi di fenotipizzazione tradizionali sono ben standardizzati e altamente riproducibili. Anche i più comuni sistemi commerciali per identificazione biochimica forniscono, in riferimento al biotipo osservato, un biotype number che può essere facilmente confrontato fra più isolati. Presentano però il grosso inconveniente di poter presentare come del tutto simili fenotipicamente isolati che potrebbero essere geneticamente diver- 
si. Tuttavia il confronto fenotipico primario tra isolati è l'unico a poter essere facilmente effettuato in tutti i laboratori di primo livello ed è ovviamente poco costoso. Il limite più ovvio ad un simile tipo di approccio è che il fenotipo di un microrganismo è il risultato della espressione di solo alcuni dei suoi geni, non di tutti e non ovviamente di quelli più conservati che hanno, al contrario, una rilevanza primaria nel discriminare genotipicamente fenotipi tra loro simili se non identici. Talora un particolare fenotipo osservato può essere sufficientemente specifico per confermare un outbreak, altre volte il fenotipo può essere così comune da non confermare con ragionevole certezza il sospetto epidemiologico: es.: ceppi di A. baumannii MDR richiedono la genotipizzazione per un corretto inquadramento dell'outbreak.

\section{I metodi di tipizzazione genotipici}

Questo tipo di approccio consente mediamente uno studio molto più specifico e discriminante degli isolati coinvolti in cluster o eventi epidemici e si basa sullo studio del genoma dei microrganismi essenzialmente il loro DNA. Alcuni metodi analizzano il genoma nella sua interezza; altri invece concentrano su regioni conservate quali, ad esempio per le Enterobacteriaceae, le regioni ERIC Enteric Repetitive Intergenic Consensus o le unità REP Repetitive Extragenic Palindromic units; altri ancora utilizzano l'analisi di geni housekeeping geni costitutivi altamente espressi.

Le tecniche di biologia molecolare hanno rivoluzionato la potenzialità dei laboratori di microbiologia, essendo caratterizzate da una notevole applicabilità a specie diverse e da un elevato potere discriminante. La genotipizzazione dimostra definitivamente il grado di relazione clonale tra due o più isolati della stessa specie. Questi metodi tuttavia richiedono, in genere, apparecchiature sofisticate e costose e, soprattutto, uno staff addetto ben addestrato. Non esiste, inoltre, un sistema comune in grado di tipizzare tutte le specie microbiche, il metodo deve essere scelto ed adattato a seconda del principio di funzionamento e delle caratteristiche genomiche della specie in esame e, non ultima, in base all'expertise del laboratorio, con la consapevolezza che alcune metodiche sono caratterizzate da una bassa riproducibilità soprattutto inter-laboratorio. Ciò premesso è indubbio che $\mathrm{i}$ metodi di tipizzazione microbica siano diventati parte integrante dei laboratori di microbiologia clinica e sono peraltro piuttosto numerosi. Fra questi occorre ricordare:

- Pulsed field gel electrophoresis PFGE,

- Restriction fragment length polymorphisms RFLP,
- Ribotyping,

- Restriction fragment length polymorphism studies e Randomly amplified polymorphism DNA RAPD,

- Amplified fragment length polymorphism AFLP e Fluorescent amplified fragment length polymorphism f-AFLP,

- Repetitive sequence-based PCR REP-PCR

- Multiple-locus variable number of tandem repeats VNTR analysis MLVA

- Multilocus sequence typing MLST,

- spa-Typing,

- Comparative genomic hybridisation,

- Whole genome sequencing WGS,

- Tipizzazione molecolare: metodi MALDI TOF-based.

La scelta del metodo di tipizzazione non è facile anche se peculiarità e limitazioni delle varie metodiche sono ormai abbastanza definite, fra queste ad esempio:

- la MLST è costosa e molto laboriosa;

- la PFGE soffre di uno scarso potere di risoluzione delle bande di dimensioni simili e ha, scarsa riproducibilità inter e intra laboratorio, scarsamente trasferibile tra laboratori è, inoltre, un metodo che richiede tempi lunghi;

- la AFLP fornisce un profilo completo, ma complesso, del DNA genomico di un isolato, che può essere poi molto difficile da interpretare;

- la REP-PCR utilizza primers che hanno come bersaglio sequenze ripetute intergeniche lungo il DNA ed è caratterizzata da un elevato potere discriminante, tanto da essere nata per una caratterizzazione di sub specie.

Essenzialmente i metodi di tipizzazione devono poter consentire di confermare o escludere la relazione clonale fra isolati coinvolti in focolai epidemici e permettere di conservare il database degli isolati caratterizzati nel tempo al fine di costruire all'occorrenza il percorso filogenetico compiuto da un clone.

Tutti i metodi devono poter consentire uscite grafiche di facile interpretazione, quali: il dendrogramma, o albero filogenetico, la matrice di similarità, scatter plot che in genere originano dal PCA analysis.

Tali uscite nel loro insieme costituiscono la rappresentazione grafica, in cui generalmente sull'asse delle ascisse sarà così espressa la distanza logica dei clusters secondo la metrica definita e sull'asse delle ordinate il livello gerarchico di aggregazione $(5,15,35,29)$.

Sono di grande impatto visivo, e consentono di raggruppare $o$, in termine tecnico, clusterizzare in forma grafica $i$ valori di similarità ottenuti tra $i$ vari isolati a confronto. 


\section{Pulsed field gel electrophoresis PFGE}

Consente la separazione di grandi molecole di DNA fino a $10 \mathrm{Mb}$ mediante l'elettroforesi su gel tipicamente gel di agarosio. La possibilità di separare molecole di così grandi dimensioni si realizza grazie ad una tecnica di elettroforesi tradizionale in cui però la direzione del campo elettrico applicato al DNA viene periodicamente alternata in intensità e direzione. Viene utilizzata per classificare i batteri oltre il livello di specie basandosi su siti di restrizione all'interno del genoma batterico. Il processo prevede l'incorporamento dell'organismo in cubetti di agarosio, lisi dell'organismo in situ, e digestione del DNA genomico con una endonucleasi di restrizione che riconosce sequenze specifiche del genoma batterico; tipicamente si producono 10-20 frammenti di DNA. Poiché questi frammenti sono troppo grandi per essere separati su gel convenzionale si ricorre all'uso di un sistema di tensione alternata noto come elettroforesi a campo pulsato grazie al quale i frammenti vengono differenziati sulla base della loro dimensione molecolare.

Il grado di relazione genetica fra gli isolati si ottiene poi comparando per numero e dimensione $\mathrm{i}$ frammenti ottenuti. Il pattern di un isolato viene definito come pulso-tipo. Alla base della comparazione di due o più pulso-tipi vi può essere una semplice elaborazione manuale, che in genere si basa sull'applicazione delle regole di Tenover secondo le quali due pulso-tipi che differiscono per un numero di bande compreso fra 0 e 3 indica germi in relazione clonale; da 3 a 7 indica germi fra loro correlati ma non clonali; oltre 7 bande di differenza indicano germi simili ma non geneticamente correlati. È anche possibile l'elaborazione con il supporto di software dedicati, il più acclarato dei quali è sicuramente BioNumerics. Esistono poi siti web in cui è possibile elaborare i pulso-tipi ottenuti e compararli con altri presenti ad es http://www.cdc.gov/pulsenet.

Come sopra ricordato il maggior problema della PFGE, da molti ritenuta il gold standard del fingerprinting, è la trasferibilità fra laboratori, è una tecnica piuttosto laboriosa e spesso caratterizzata da uno scarso potere discriminante, oltre che da una estrema soggettività nell'interpretazione dei risultati (29). Lo stesso CDC nel suo Pulsenet descrive le seguenti limitazioni:

- richiede molto tempo e un alto livello di abilità degli operatori

- non è adatta allo studio di tutti i cloni

- i pattern che si ottengono variano molto in relazione alla manualità

- è difficile ottimizzare le condizioni di migrazione in ogni zona del gel

- le bande non corrispondono esattamente alle sequenze, quindi non è possibile sapere se bande della stessa dimensione rappresentino, in isolati diversi, lo stesso frammento di DNA. A suo vantaggio i costi relativamente contenuti mediamente 20-30 euro a isolato ed il fatto che essa rappresenti comunque un approccio preliminare per la gran parte delle specie microbiche nell'intento di valutare una macrorestrizione preliminare dei frammenti del DNA genomico dei isoalti in esame (29).

\section{Restriction Fragment Length Polymorphisms RFLP}

È stata una delle prime tecniche ad essere ampiamente utilizzata per evidenziare le variazioni a livello di sequenza di DNA. Il principio su cui si fonda è il confronto dei profili di bande ottenute dopo la digestione enzimatica del DNA. Gli enzimi che si utilizzano sono gli enzimi di restrizione che, come noto, sono in grado di riconoscere sequenze specifiche siti di restrizione lungo un filamento di DNA.

Le mutazioni anche puntiformi che possono essere intervenute in una linea clonale nei siti di restrizione si evidenziano con possibili modifiche dei profili di restrizione del DNA cromosomiale. L'effetto della mutazione può essere la soppressione o la formazione di un sito di restrizione che, a caduta, causerà una diversa ripartizione in frammenti del genoma del clone in esame originando, all'elettroforesi in gel, bande di lunghezza diversa rispetto all'atteso. Con la comparazione delle bande ottenute si può definire il grado di relazione genetica degli isolati. Nel caso in cui la digestione del DNA dovesse fornire una sequenza continua di frammenti smear, il trasferimento su una membrana di nitrocellulosa e la successiva ibridizzazione con sonde specifiche Southern blotting permetteranno di risolvere il problema. Tali sonde sono scelte in relazione all'informazione che si vuole ottenere e in genere derivano dalla marcatura del DNA di un ceppo preso come riferimento nei confronti del quale si vuole analizzare il ceppo in esame. A seconda che il metodo di marcatura utilizzato sia "caldo" ossia con isotopi radioattivi o "freddo" es.: chemiluminescenza la visualizzazione avverrà su di una lastra per autoradiografia o su un diverso supporto. Il risultato sostanzialmente è il medesimo ossia una sequenza di bande su un supporto solido. La RFLP è una tecnica molto laboriosa soprattutto nella versione che utilizza il Southern blotting che richiede tempi lunghi, una grande manualità degli operatori e, inoltre, una grande quantità di DNA campione; questi fattori limitanti spesso poco si conciliano con l'esigenza di tempestività di una indagine finalizzata al controllo di un outbreak (24). 


\section{Ribotyping}

I tre geni codificanti le molecole di rRNA 16S, $23 \mathrm{~S}$ e $5 \mathrm{~S}$ sono contenuti nell'operone ribosomiale, ove il numero di copie dei geni variabile da specie a specie, la loro sequenza nucleotidica e le dimensioni stesse dell'operone sono altamente conservate all'interno di una specie batterica (22). Poiché il gene $16 \mathrm{~S}$ rRNA è il più conservato dei tre geni, il suo sequenziamento costituisce ancora il gold standard per l'identificazione e la classificazione tassonomica delle specie batteriche (17, 27). L'utilizzo della ribotipizzazione, ossia della tipizzazione microbica basata sullo studio del $16 \mathrm{~S}$ rRNA fu introdotta Grimont e Grimont (10). Il concetto su cui si fonda è sostanzialmente simile a quelli fin qui descritti, ossia si parte dal genoma del microorganismo in studio, lo si sottopone a restrizione mediante specifici enzimi, si ottengono diversi frammenti che vengono poi separati mediante elettroforesi su gel. Gli isolati avranno pattern specifici in base ai quali sarà possibile la comparazione. Da quando fu descritto per la prima volta nel 1986 si contano più di 2000 pubblicazioni e riguardano in totale più di 200 generi batterici e fungini (10). Il termine "ribotipizzazione" è termine improprio, che porta a un equivoco ossia che i polimorfismi osservati derivino direttamente dalle sequenze dell'rRNA.

Si tratta, in realtà, del polimorfismo del DNA che si riflette sulla Restriction Fragment Length Polymorphisms RFLPs di geni housekeeping $\mathrm{i}$ quali tipicamente affiancano quelli codificanti l'rRNA. Tali geni sono sottoposti a minore ricombinazione sono definiti quiescenti dal punto di vista ricombinazionale ed essendo più stabili meglio si adattano agli studi di filogenesi e tipizzazione (18). Essendo il metodo basato sull'identificazione di frammenti immediatamente a monte e a valle di ogni operone ribosomiale il numero di questi operoni che è variabile nelle diverse specie, ma costante all'interno della stessa specie determinerà il numero di bande del ribotipo e quindi la sensibilità del metodo. Il ribotyping è consigliato solo per specie microbiche che abbiano almeno quattro operoni ribosomiali all'interno del loro genoma. Anche questo metodo è applicabile su varie piattaforme automatizzate. Una variante è costituita dalla PCR Ribotyping, sviluppata nel $1990(11,18)$ al fine di agevolare, sia in termini di tempo sia mediante l'eliminazione di sonde, le tecniche di tipizzazione. Sebbene molto utilizzata questa metodica presenta tuttavia, in entrambe le versioni, alcune limitazioni d'uso e non può essere considerata come universalmente applicabile (3). Inoltre la PCR ribotyping rappresenta un eccellente sistema d'identificazione microbica a livello di specie, ma non può essere considerata alla stessa stregua per la tipizzazione clonale $(3,8$, $12,19,23)$. Un'alternativa alla PCR ribotyping è costituita dalla ARDRA Amplified rRNA gene Restriction Analysis basata su una reazione di amplificazione nel 16S rRNA gene seguita da una restrizione. Questa metodica è stata sviluppata per tipizzare a livello di subspecie lo Streptococcus uberis e per le sue peculiarità è sicuramente più efficace del ribotyping nell'evidenziare le variazioni intraspecie (14).

\section{Random Amplification of Polymorphic DNA RAPD}

In realtà il metodo si classifica nel più generico $\mathrm{e}$ vasto gruppo definito come Arbitrary amplification of polymorphic DNA sequences che nel corso degli anni si è arricchito di diverse varianti sul tema, tutte, comunque, basate sull'amplificazione preventiva di frammenti e tutte destinate alla caratterizzazione genetica dei microrganismi. Appartengono a questo gruppo la Arbitrarily Primed PCR AP-PCR e la Random Amplified Polymorphic DNA RAPD analysis $(30,32)$. Questi metodi utilizzano approcci completamente differenti, ma la base teorica è la stessa: sequenze arbitrarie di DNA vengono utilizzate come primer in genere di circa 10-12 paia di basi che hanno come bersaglio una sequenza genomica. L'amplificazione è condotta a basse temperature di annealing e questo consente i mismatch, cioè permette ai primer di formare sia legami specifici che aspecifici da cui l'espressione arbitrarily. $\mathrm{Si}$ ottengono, anche in questo caso, frammenti che identificano un profilo genetico. Almeno in linea teorica i "primer arbitrari" generano pattern di amplificazione sufficienti per stabilire le relazioni clonali fra isolati di una stessa specie ed è altresì comunemente accettato che qualunque polimorfismo osservato nel confronto fra microrganismi negli ampliconi ottenuti rappresenti il risultato di mutazioni genetiche puntiformi, di delezioni o di inserzioni di elementi genetici con conseguente alterazione del sito di priming. In sostanza, gli stessi meccanismi genetici che erano alla base dell'origine di bande di diversa lunghezza nei metodi descritti in precedenza, sono alla base anche dell'ottenimento di ampliconi di diversa dimensione nei metodi basati però sull'amplificazione. Quindi il primo vantaggio nell'utilizzo di questi sistemi risiede nel fatto che sono sufficienti quantità di DNA sensibilmente inferiori ai metodi precedenti, quindi, è possibile partire anche da una sola colonia dell'isolato in questione. Questo comporta una minore manualità e minor tempo necessario per il confronto di più isolati. Tuttavia, è segnalata da più autori la possibilità che prodotti generati da loci diversi di spe- 
cie non correlate possano co-migrare generando pattern di bande apparentemente simili quindi la similarità fra microrganismi, in realtà, non correlati può essere sovrastimata (30). Sebbene questi metodi siano di solito considerati validi nella rapida discriminazione di isolati e nell'identificazione di outbreaks, tale criticità, unita alla scarsa riproducibilità intra ed inter-laboratorio a causa soprattutto di protocolli di amplificazione arbitrariamente adattati dall'operatore alla propria strumentazione, fa si che l'attendibilità delle informazioni prodotte lasci molto a desiderare (29).

I risultati possono inoltre essere influenzati da diversi fattori tra cui: la concentrazione dei sali di Magnesio $(\mathrm{Mg}++)$, il metodo di estrazione del DNA, le variazioni batch-to-batch nella sintesi dei primers, il rapporto DNA template-concentrazione dei primers molti operatori tendono a lavorare con eccessive concentrazioni di primers, il tipo di termociclatore usato, il tipo e la concentrazione di Taq polimerasi usata. Per questa ragione deve essere usata molta cautela nell'uso dei risultati forniti da questo sistema, soprattutto se si intende confrontare i dati tra diversi laboratori.

Amplified Fragment Length Polymorphism AFLP e Fluorescent Amplified Fragment Length Polymorphism f-AFLP

La PCR-AFLP o più semplicemente AFLP fu sviluppata nei primi anni 1990 da Keygene, (16, 35). Utilizza enzimi di restrizione per digerire il DNA genomico, seguita da una reazione di ligation con adattatori per le estremità adesive originate su frammenti di DNA genomico frammenti di restrizione ottenuti mediante la restrizione degli enzimi medesimi. Segue una successiva fase di amplificazione selettiva, che utilizza primer le cui regioni target consistono proprio nelle sequenze degli adattatori per questo si parla di amplificazione selettiva. Alla fine delle reazioni di restrizione, ligasi ed amplificazione si ottiene una serie di ampliconi rappresentativi dell'intero genoma microbico. I frammenti amplificati vengono poi separati mediante elettroforesi capillare, in genere utilizzando sequenziatori a capillare.

La rivelazione dei frammenti può essere radiometrica se vengono usati nucleotidi radio marcati nella reazione di amplificazione ovvero fluorimetrica se si utilizzano nucleotidi marcati con fluorocromi f-AFLP. Si otterranno degli elettroferogrammi in cui ogni picco sarà rappresentativo di un frammento amplificato avente una determinata lunghezza. Anche in questo caso il confronto fra due e più isolati consisterà nella comparazione dei picchi comuni e/o dei picchi assenti. La AFLP è dotata di una eccellente potere discriminante, ma presenta diversi svantaggi:
- richiede personale particolarmente addestrato, soprattutto nell'uso dei sequenziatori automatici e delle procedure di biologia molecolare

- le apparecchiature dedicate sono costose

- comporta tempi lunghi almeno tre giorni

- offre una scarsa uscita grafica

Quest'ultima rappresenta, forse, l'aspetto più penalizzante in quanto non esistono software dedicati che consentano di convertire direttamente $\mathrm{i}$ picchi ottenuti da due o più isolati in matrici di similarità $o$ in dendrogrammi. È necessaria un'elaborazione per lo più manuale dei risultati utilizzando parametri quali l'indice di Dice che esprime il grado di somiglianza fra due o più isolati e si calcola confrontando le bande in questo caso gli ampliconi comuni fra i microrganismi a confronto. Per un'interpretazione sommaria un indice di Dice tra 1 e 0.9 indica una relazione clonale fra due isolati; tra 0.9 e 0.7 indica correlazione ma non clonalità; tra 0.7 e 0.6 indica isolati simili ma non correrelati; un valore inferiore a 0.6 indica isolati non correlati.

\section{Repetitive Extragenic Palindromic Sequence- PCR REP-PCR}

La REP-PCR prevede una fase di amplificazione in cui il target genomico è rappresentato da regioni ripetute altamente conservate specie-specifiche repetitive DNA sequences presenti nel genoma della maggior parte di germi Gram negativi ed in molti Gram positivi. Sono state identificate tre famiglie di sequenze ripetute che includono: 1 frammenti di circa 35-40 bp Repetitive Extragenic Palindromic REP sequence; 2 frammenti di 124127 bp Enterobacterial Repetitive Intergenic Consensus ERIC sequence; 3 frammenti di $154 \mathrm{bp}$ BOX element. Queste sequenze sono localizzate come regioni intercalate fra i geni lungo l'intero genoma. Possono essere presenti in entrambi gli orientamenti ed i primer per le reazioni di amplificazione sono stati disegnati in modo tale da condurre la reazione di amplificazione verso l'esterno delle sequenze ripetute ed invertite per REP ed ERIC $(21,34)$. Alla reazione di amplificazione, selettiva per regioni specifiche del genoma localizzate tra le sequenze REP, ERIC e BOX, può essere fatta seguire la risoluzione degli ampliconi mediante elettroforesi su gel. I pattern ottenuti possono essere comparati e clusterizzati utilizzando programmi quali: BioNumerics Applied Maths NV, Austin, Texas, USA, AMBIS system Scanalytics, Waltham, MA, USA e GelCompar Applied Maths, Kortrijk, Belgium (33). La REPPCR è stata automatizzata su un sistema automatico, il DiversiLab bioMérieux Italia, Firenze Italy, che sfrutta la tecnologia della microelettroforesi capillare su microchip per la separazione 
degli ampliconi ottenuti e che consente una notevole semplicità d'uso con ampie possibilità di elaborazione, archiviazione e recupero dei dati.

Il sistema offre la possibilità di produrre grafici in più formati matrici di similarità, dendrogrammi, ecc che consentono sia una "clusterizzazione" raggruppamento di tipo gerarchico degli isolati, con la metodologia Unweighted Pair Group Method UPGMA, sia una "clusterizzazione" non gerarchica mediante Principal Components Analysis PCA. Quest'ultima consente di visualizzare graficamente la vicinanza o distanza genetica fra gli isolati oggetto del fingerprinting (34). DiversiLab utilizza un software web-based, per cui è possibile confrontare i propri fingerprinting anche con altri presenti nel web, e consente la gerarchizzazione degli isolati sulla base di due diversi coefficienti di similarità opzionabili dall'operatore:

$\Rightarrow$ Pearson Correlation (PC): che attribuisce maggiore rilievo all'intensità delle bande ottenute piuttosto che alla loro presenza o assenza; $\Rightarrow$ Kullback-Leibler (KL): che valorizza, al contrario, proprio la presenza o assenza delle bande.

I grandi vantaggi della REP-PCR, dimostrati dal suo massivo utilizzo, consistono nella facilità esecutiva soprattutto per la versione automatizzata, nella facile interpretabilità sono peraltro possibili diverse uscite grafiche, nella elevata riproducibilità, nella estensiva applicabilità può essere applicata ad un ampio numero di specie $(6,13,29)$.

Gli svantaggi sono pochi e consistono nella limitazione d'uso legata alla possibile presenza di un numero poco rappresentativo di sequenze REP eventualità molto remota, tanto che l'uso della REP-PCR sta trovando grande applicazione anche per i miceti ed al dover necessariamente partire dalla colonia. È dimostrato come sia poco utile, se non addirittura fuorviante, nella tipizzazione degli MRSA (29).

È anche uno dei pochi metodi per i quali è possibile avere una stima dei costi realistica: circa 20 euro a isolato (29).

\section{Multiple-locus variable number of tandem repeats VNTR analysis MLVA}

$\grave{E}$ un metodo utilizzato per eseguire la tipizzazione molecolare di microrganismi particolari. Utilizza la variazione naturale del numero di sequenze di DNA ripetute in tandem ossia sequenze di due o più nucleotidi ripetute una di seguito all'altra, es.: CTTCG CTTCG CTTCG CTTCG, collocate nel genoma di una varietà di organismi. Il metodo proviene dalla scienza forense dove viene utilizzato per il DNA fingerprinting in campioni di origine umana. I profili di tipizzazione molecolare vengono utilizzati per studiare le vie di trasmissione, per valutare le fonti di infezione e anche per valutare l'impatto di varie forme d'intervento umano, quali le vaccinazioni e l'uso di antibiotici, sulla composizione delle popolazioni batteriche. Fonda la sua essenza sul fondamento che il genoma dei procarioti contiene una vasta gamma di repetitive DNA elements che vanno dal singolo nucleotide che si ripete a decine di nucleotidi. Variable-number tandem repeats VNTRs sono ripetizioni che si trovano in tandem a dimostrare la variabilità tra ceppi. La MLVA si è ormai dimostrata affidabile per la tipizzazione clonale in molte specie microbiche tra cui A. baumannii.

Il principio della tipizzazione molecolare con VNTRs è che questi elementi possono mutare, creando diversi alleli dello stesso locus VNTR TR si possono generare per varie ragioni per esempio per slittamento e mispairing durante la replicazione del DNA a causa della dissociazione occasionali DNA polimerasi. Il targeting di questi elementi, che spesso variano nel numero tra i diversi ceppi della stessa specie la definizione di un variabile numero TR (VNTR), è stato utilizzato con successo per discriminare tra ceppi di procarioti. La MVLA si basa sull'esecuzione di PCRs dei loci VNTR seguito da accurato dimensionamento dei prodotti di PCR mediante uso di sequenziatore di DNA automatizzato che sfrutta le proprietà dell'elettroforesi capillare per l'analisi dei frammenti. La dimensione del prodotto ottenuto viene utilizzata per calcolare il numero di unità ripetute in ogni locus. Il numero di ripetizioni calcolate per i loci VNTR alleli sono combinate in una stringa che consiste ad esempio numeri interi 140-2-4-1-7-1-6 e viene indicato come il profilo MLVA. Ogni profilo MLVA è unico. Il profilo MLVA può essere utilizzato per il confronto e per lo studio dei cluster. I valori ottenuti possono essere facilmente comparati con un database centrale disponibile sul web; un sito: http://www.mlva.net/ (26). Esempi di applicazioni di MVLA sono quelli su STEC O157 e S. enterica sierotipo Typhimurium. Anche questa dotata di un eccellente potere discriminante, è tuttavia molto laboriosa e costosa, soffre nella riproducibilità ed è destinata più alla epidemiologia globale che a quella locale. Altra importante limitazione intrinseca del metodo è che MLVA non è un metodo "universale", questo perché la scelta dei primers deve essere calzata sulla singola specie di patogeni in osservazione (29). Non è possibile per la natura del metodo una stima puntuale dei costi, che tuttavia, si mantengono elevati.

\section{Multi Locus Sequence Typing MLST}

Questa metodica ha offerto un nuovo approccio 
alla epidemiologia molecolare essendo in grado di identificare e tracciare la diffusione globale dei ceppi microbici mediante l'uso di Internet. Presenta il grande vantaggio di consentire lo studio dell'epidemiologia globale riuscendo a caratterizzare un vasto numero di genotipi attraverso l'analisi di variazioni genetiche che si accumulano lentamente. La combinazione di potere discriminante e stabilità clonale della MLST ha dimostrato essere estremamente efficace nell'identificare i diversi cloni che si possono originare in una popolazione batterica ed ha anche fornito gran parte delle nostre conoscenze in campo di genetica delle popolazioni. Con l'utilizzo di una sola coppia di primer vengono sequenziati, su entrambi i filamenti di DNA, frammenti interni delle dimensioni di 450-500-bp appartenenti a sette geni housekeeping ottenendo, per la maggior parte dei batteri patogeni, informazioni sulle variazioni sufficienti ad identificare alleli diversi di una popolazione. Le differenze nella sequenza definiscono un diverso allele e i diversi alleli, per ognuno dei sette geni studiati, forniscono un "profilo allelico" che in maniera inequivocabile definisce il sequence type ST di ogni isolato. Sequenze che differiscono anche per un singolo nucleotide sono assegnate come alleli diversi. Il grande numero di alleli possibili per ognuno dei sette loci è alla base della capacità di distinguere miliardi di differenti profili allelici. Una media di 30 alleli per locus, ad esempio, permette di distinguere circa 20 miliardi di genotipi diversi.

Un ulteriore vantaggio della MLST è che i profili allelici possono essere ottenuti non solo dagli isolati ma anche da materiale clinico mediante amplificazione PCR dei sette geni housekeeping direttamente dal CSF o sangue. Così il germe responsabile del processo infettivo può essere caratterizzato anche quando non riesce ad essere coltivato dal materiale clinico. La MLST consente la generazione di coded-types ST ed ha un database facilmente accessibile http://www.mlst.net/ ma è costosa e per sua natura più indicata a studi genetici di popolazione piuttosto che alla tipizzazione. Attualmente nel sito sono disponibili gli ST e le relative analisi comparative per numerose specie microbiche (4). La limitazione del metodo rimane legata alla sua notevole laboriosità nonché ai costi ancora molto elevati mediamente 50 euro a isolato (29).

\section{spa-Typing}

È il metodo più noto della Single Locus Sequence Typing SLST ed è elettivamente utilizzato per la tipizzazione di $S$. aureus applicato in modo particolare alla proteina A. Esso comporta il sequenziamento della regione polimorfica X della proteina A di S. aureus. Rappresenta anche un metodo storico, in quanto il sequenziamento della proteina A, finalizzato allo studio dei polimorfismi, è stato la prima applicazione di tipizzazione mediante sequenziamento. La notevole diversità nelle regioni VTNR del gene spa si riflette non solo in un numero variabile di short repeats $24 \mathrm{bp}$, ma anche in varie mutazioni puntiformi. Ad ogni repeats viene associato un codice ed lo spa-type è dedotto dall'ordine dei singoli repeats. Sebbene lo spa-typing possieda un potere discriminante, che globalmente è inferiore alla PFGE, è un approccio efficace, semplice da usare, rapido, poco costoso è dotato di eccellente riproducibilità ed è caratterizzato da un'eccellente uscita interpretativa soprattutto se si utilizza il software StaphType. Altro vantaggio è la totale "trasferibilità e confrontabilità" dei dati soprattutto usando il database Ridom http://spaserver.ridom.de. Infatti, i dati possono essere caricati su spa server confrontati e condivisi con tutti gli utilizzatori. Questo ha reso, ad oggi, lo spa database il più grande database riguardante la tipizzazione di $S$. aureus dati da più di 200 laboratori, dislocati in più di 75 nazioni. Il database è aperto, dinamico e controllato qualitativamente. Tuttavia, anche questo metodo non è immune da criticità e limitazioni. La principale limitazione risiede proprio nel fatto che si fonda su un typing di un singolo locus genico, pertanto, può classificare in maniera impropria alcuni tipi a causa di eventi ricombinativi che possono essersi verificati (31). Cioè è dotato di potere discriminatorio insufficiente soprattutto laddove un clone o un piccolo numero di cloni sono divenuti endemici non è, inoltre, raccomandato per piccole realtà ospedaliere (31).

\section{Comparative genomic hybridisation}

Si fonda sulla tecnologia del DNA microarray, utilizzata principalmente negli studi di tipizzazione. Sonde specifiche sono adese su una superficie solida, tali supporti cimentati con il campione/isolato batterico contenente il DNA target consentiranno l'individuazione di sequenze complementari. Pertanto, i microarrays rappresentano un dispositivo di facile uso per la ricerca di geni sia che siano marcatori di resistenza sia che si tratti di markers specifici di specie, o che si tratti di sonde in grado di rilevare varianti alleliche di una specifico gene. Le sonde sul microarray possono essere sia ampliconi di 200 bp che oligonucleotidi $>70$ mers. In relazione al numero di sonde adese sulla superficie solida si possono distingure i microarrays in: a low-density centinaia di sonde, b highdensity da centinaia a migliaia di sonde. Nella sua applicazione più diffusa e comune, il DNA totale estratto da un patogeno oggetto dello studio, viene marcato sia chimicamente sia attraverso reazione 
enzimatica ed ibridizzato con le sonde presenti nel microarray. Il DNA non legato è rimosso, mentre quello legato in maniera stabile con le sonde presenti sulla matrice, viene misurato mediante uno scanner. I dati sono quindi analizzati da un software in grado di attribuire specifici segnali di positività ai marcatori di resistenza, alla identificazione microbica, o alla tipizzazione se trattasi di sonde in grado di rilevare la variabilità intraspecie. In particolare, quest'ultimo aspetto ha trovato un discreto sviluppo ed applicazione nell'analisi di mutazioni genetiche come "single-nucleotide polymorphisms SNPs". In particolare, questa tecnologia, soprattutto nella versione high-density consente un approccio/studio dell'intero genoma, tanto che ha, per esempio, reso possibile l'identificazione di $10 \mathrm{~S}$. aureus lineages maggiori, responsabili della maggior parte delle infezioni umane (29). Alcuni esempi di applicazione eloquente di high-density arrays: Sam-62 basato su 62 S. aureus whole genome sequencing WGS e su ben 153 sequenze plasmidiche, ha permesso di dimostrare la trasmissione di MRSA non individuati da altre metodiche.

Affymetrix DNA microarray ossia una piattaforma basata sulle ORFs Open Reading Frames identificate in 31 cromosomi e 46 plasmidi di isolati di E. coli e Shigella, ha consentito di determinare rapidamente la presenza/assenza di geni di virulenza negli $E$. coli O104:H4 recentemente noti alla cronaca come causa di un evento epidemico. Questi, sono entrambi esempi importanti di come il Whole Genome Microarray possa essere una valida alternativa al classico Whole Genome Sequencing WGS, consentendo un approccio più rapido, meno costoso, ma ugualmente attendibile ai fini dello studio in tempo reale di un evento epidemico (29). Tuttavia, anche questa metologia, sta incontrando delle difficoltà nell'uso estensivo nei laboratori, per via dei costi, ancora troppo elevati mediamente superiori ai 100 euro a campione.

La "Alere Technologies" ha recentemente sviluppato un sistema microarray in microstrip, adatto sia alla diagnostica che agli studi epidemiologici, in grado di testare da 8 a 96 campioni: Alere StaphyType DNA microarray per S. aureus copre 334 sequenze target 170 geni distinti e loro varianti. I risultati dell'analisi, così come l'attribuzione degli isolati di $S$. aureus ad un particolare lineages, vengono condotte automaticamente sulla base dei profili di ibridizzazione evidenziati. Grazie alla tecnologia introdotta i costi sono scesi ai da 20-30 euro/isolato costo comparabile alla PFGE (29). Alere ha, inoltre, recentemente sviluppato altri applicativi per: E. coli, P. aeruginosa, L. pneumophila, e Chlamydia trachomatis.

I dati ad oggi disponibili sembrano dimostrare una elevata efficienza del microarray, tuttavia, è imperativa la necessaria prudenza, prima di un suo uso estensivo, soprattutto è necessario indagare più a fondo la riproducibilità intra ed interlaboratorio. Una limitazione di questo approccio, soprattutto della versione "whole genome microarray" e' che al metodo sfuggono sequenze non incluse nelle sonde presenti sul supporto solido del microarray.

\section{Whole Genome Sequencing WGS}

Il sequenziamento nella sua più recente accezione, ossia il Next Generation Sequencing NGS o altrimenti noto come sequenziamento di seconda generazione ovvero come High-Throughput Sequencing HTS ha consentito di trasformare questa tecnologia in un metodo di studio delle variazioni genomiche costo-efficace. Il termine next generation riferisce alla transizione del sequenziamento dal metodo Sanger all'attuale.

Il vantaggio evidente del NGS rispetto al "Sanger sequencing" è la capacità di generare milioni di letture ciascuna di 35-700bp in una singola corsa a costi comparabili con il vecchio metodo.

Per arrivare a costruire la completa analisi nucleotidica di un genoma, occorre analizzare ed assemblare nelle regioni di overlapping - assemblaggio de novo multipli di sequenze corte. Il WGS è divenuto un approccio potente ed estremamente interessante finalizzato anche agli studi di epidemiologia (29). È molto probabile che in un futuro, non molto lontano, questa tecnologia trovi ampio spazio nella clinica permettendo un'accurata identificazione e caratterizzazione degli isolati batterici e fungini e nello studio della relazione clonale in caso di epidemie. La relazione genetica fra isolati batterici o fungini può, infatti, essere determinata anche comparando i genomi nella loro interezza con un potere di risoluzione/discriminazione molto elevato in teoria anche a livello di singolo nucleotide. Tuttavia, per quanto attraente al momento questa prospettiva è lontana dalla applicazione pratica estensiva.

Oltre alle applicazioni epidemiologiche la WGS può essere di aiuto nella definizione della caratteristiche fenotipiche sia di virulenza che di antibiotico resistenza dei patogeni. Il primo tentativo di creare "un resistoma artificiale" è stato dimostrato comparando un gene predittivo della resistenza con i risultati del test di sensibilità antimicrobica tradizionale, allo stesso modo si può operare con il "taxoma" gene per tossina vs espressione fenotipica.

La vera sfida, tuttavia, non è produrre sequenze, ma elaborarle ed interpretarle rapidamente mediante comparazione con un data set appropriato. Idealmente, queste informazioni dovrebbero includere, e quindi consentire, anche un confron- 
to diretto con i risultati ottenuti con metodi di tipizzazione convenzionali ad esempio, PFGE, MLST, e dovrebbero essere conservati in banche dati accessibili a livello globale. Tuttavia, le sequenze ottenute in NGS sono relativamente brevi, e questo è un ostacolo all'assemblaggio de novo. Pertanto, in realtà, quando parliamo di WGS ci riferiamo nella pratica a circa il $90 \%$ dell'intero genoma. I gaps tra regioni assemblate contigue è causato soprattutto dalla presenza di ripetizioni disperse o in tandem, le attuali piattaforme NGS non sono in grado di risolvere in maniera eccellente tali VNTRs.

Per migliorare l'assemblaggio de novo sono quindi necessarie nuove piattaforme in grado di processare ed assemblare sequenze più lunghe. Recentemente si è anche iniziato a parlare di "sequenziamento di terza generazione" PacBio lanciato dalla Pacific Biosciences, che è in grado di produrre sequenze molto lunghe lunghezza media di 2-3 kb fino ad un massimo di $7 \mathrm{~kb}$. Le "nanopore sequencing technologies" sviluppata da Oxford Nanopore sembrerebbe essere in grado di spingersi fino alle $100 \mathrm{~kb}$.

Per dare un'idea degli sviluppi in questa direzione il progetto " $100 \mathrm{~K}$ Genome Project", che è una iniziativa della Food and Drug Administration FDA, di Agilent, e della Università della California, ossia organi federali assieme a partners privati che si sono dati lo scopo di sequenziare 100.000 genomi di altrettanti patogeni alimentari nei prossimi 5 anni.http://100kgenome.vetmed.ucdavis.edu.

Il principale limite di questo sequenziamento è il costo decisamente proibitivo e la percentuale di errore ancora molto elevata circa il $15 \%$. I costi stimati della NGS si aggirano attorno ai 100-150 USD per isolato circa 75-110 euro, ed includono la preparazione del campione, la reazione di sequenza la sua analisi ed il confronto in banca dati. Altro aspetto apprezzabile è la configurazione della strumentazione, sempre più compatta e di ridotte dimensioni i cosiddetti desktop sequencer o bench top che ne rende possibile l'installazione nei comuni laboratori, ma anche in questo caso, va detto che i costi della strumentazione sono ancora piuttosto elevati circa 130.000 euro $(20,29)$.

In commercio esistono già dal 2010, con integrazioni e novità importanti dello scorso novembre 2012 chip semiconduttori, sequenziatori compatti che hanno reso il sequenziamento totale del genoma più accessibile. Tre sono gli strumenti più diffusi: 454 GS Junior Roche, MiSeq Illumina, Ion Torrent PGM Life Technologies (20).

\section{La spettrometria di massa MALDI-TOF nella tipizzazione}

La tecnologia MALDI TOF MS è stata proposta solo da pochi anni per l'identificazione rapida degli isolati clinici ma ha già rivoluzionato i laboratori di microbiologia per la sua elevata accuratezza, la facilità d'uso e la rapidità nell'ottenimento dei risultati. Si basa essenzialmente sullo studio delle proteine ribosomiali. Il campione la colonia dell'isolato batterico viene convertito in particelle cariche o ionizzate che vengono separate in base alla differente massa dopo la ionizzazione in base al differente tempo di volo Time of Flight in un campo elettrico applicato sotto vuoto.

Dalla misura delle particelle e con la loro relativa visualizzazione grafica, viene generato uno spettro che può essere "specie specifico" consentendo, mediante comparazione dello spettro ottenuto con quelli contenuti nel database, l'identificazione dell'isolato clinico.

Essendo lo spettro proteico di una specie, e in particolare di un isolato, frutto della sua espressione genica è corretto, almeno in linea di principio, utilizzare gli spettri proteici ottenuti mediante analisi spettrometrica alla stessa stregua dei frammenti di DNA utilizzati nella tipizzazione molecolare degli isolati coinvolti in un cluster. Sono già apparsi nella letteratura diversi esempi eloquenti di questo specifico applicativo della tecnologia MALDI TOF di cui uno particolarmente interessante confronta MALDI TOF con Rep PCR mostrandone la eccellente sovrapponibilità per germi particolarmente problematici in termini di outbreak quale Acinetobacter baumannii $(9,25)$. In particolare, la raccomandazione che emerge nella pubblicazione di Mencacci, et al (25) nella valutazione degli spettri all'interno di ciascun ceppo in esame è l'importanza di stimare la varianza spettrale delle masse in varie repliche biologiche dello stesso isolato almeno 5 repliche da colture indipendenti dello stesso isolato.

È, altresì, importante acquisire tali spettri con opportune impostazioni d'intensità del laser nonché del numero di shots. Gli spettri acquisiti in questa metodologia "dedicata" possono essere salvati in sotto-database che consentiranno, quindi, un confronto più puntale degli isolati a confronto. La valutazione della somiglianza fra isolati viene condotta usando analisi tipo PCA con le diverse possibili uscite grafiche. La letteratura in questo campo si sta arricchendo di nuove esperienze (37).

Si tratta di un approccio del tutto innovativo che, nonostante i primi incoraggianti risultati, merita un periodo di studio prima di poterne affermare l'uso in equipollenza ai metodi genetici. Vero è che, considerando la diffusione della tecnologia MALDI TOF nei laboratori di microbiologia, il suo uso, potrebbe davvero rappresentare una svolta in termini di rapidità e di facilità d'uso per più 
laboratori nella definizione degli outbreak.

\section{Come, quando e perché "tipizzare"}

Pregi e difetti dei principali metodi sono stati riassunti nella Tabella 1. In questa sono state analizzate le diverse metodologie seguendo uno schema logico-matematico, ossia assegnando un punteggio oggettivo fondato su dati tangibili, tipo, il numero di passaggi delle singole procedure, la presenza assenza di software di elaborazione/confronto di dati disponibili su web, i costi, e la facilità d'uso e d'interpretazione.

Infine, nella Figura I sono invece riportati, per i principali generi/specie i metodi di tipizzazione raccomandati sulla base di evidenze scientifiche, ordinati in base al potere discriminatorio.

Questa schematizzazione può rappresentare una valida guida nella scelta del metodo di tipizzazione più adatto.

La tipizzazione non deve essere intesa come un virtuosismo del laboratorio di microbiologia, ma piuttosto come un passaggio fondamentale per comprendere, caso per caso, come si è articolata la catena di trasmissione di un microrganismo all'interno dei nostri nosocomi. La comprensione di questo fenomeno consente, quindi, attraverso l'individuazione di eventuali "criticità" di operare assieme agli "infection control team" nell'ambito dei Comitati di Studio delle Infezioni Ospedaliere CIO ed alle Direzioni Sanitarie affinchè la catena sia interrotta e si pongano rapidamente in essere tutte le necessarie misure di contenimento e prevenzione degli eventi epidemici.

\section{Riflessioni etiche}

Infine, un ultimo aspetto riguarda la necessaria riflessione etica che sta animando la comunità scientifica a questo proposito. Si tratta cioè di raggiungere un equilibrio molto delicato, nella applicazione di queste metodiche, fra ciò che è necessario nell'interesse della salute pubblica e ciò che è nel rispetto del singolo individuo. Ossia fino a dove si può spingere la scienza animata e giustificata dall'interesse per la collettività? Sebbene la scienza in questo caso si muova con lo scopo di stabilire la trasmissione di un patogeno in ambito ospedaliero, gli utilizzi che l'applicazione di simili metodiche consentono vanno anche oltre. Per citare un caso su tutti in Olanda in una infezione da HIV, la sequenza genetica del patogeno fu usata in tribunale per l'attribuzione di colpe.

$\mathrm{O}$ ancora una epidemia di Listeriosi a Toronto in Canada nel 2008, in cui il fingerprinting finì per esssere uno strumento usato in una class-action costata 27 milioni di dollari. Quindi, non è escludibile, allo stato attuale che dati di laboratorio finalizzati all'uso epidemiologico locale finiscano per essere strumenti in cause legali e addirittura class-action. Lo studio di un sospetto evento epidemico ha come finalità scientifica indiscutibile quella di stabilire, per migliorare, la via di trasmissione di un patogeno da un paziente ad un altro, ma la percezione dall'esterno del paziente e/o dei parenti è "chi ha infettato chi?". Anche quando si arriva a dimostrare la trasmissione di un patogeno attraverso il fingerprinting, questo non significa che in maniera inequivoca sia stata attribuita la responsabilità morale della trasmissione a "qualcuno", perché anche se si tratta di infezione e non di semplice colonizzazione una serie di fattori possono essere intervenuti a cagionare l'infezione per esempio la conoscenza del rischio, delle vie di trasmissione e dei mezzi per evitare la trasmissione, la messa in opera delle adeguate misure di contenimento e precauzione. Oltre all'aspetto della attribuzione della responsabilità morale esiste l'aspetto della "proprietà legale dell'isolato" e del campione da cui quel germe in un dato momento è stato isolato. Indubbiamente appartiene al paziente! Dal momento in cui, tuttavia, se ne dimostra la cross-trasmissione la proprietà è condivisa fra più individui, cioè tutti quelli coinvolti nell'evento epidemico. Questo pone anche un'altra questione, se si tratta di ottenere un consenso informato dal paziente, nell'utilizzo dei suoi isolati, come deve essere formulato tale consenso? Chi deve rilasciare tale consenso, dal momento che in un evento epidemico più individui sono coinvolti? La questione è così delicata che è stato avviato un dibattito pubblico da Dutch Municipal Health Service GGD al quale si poteva rispondere inviando le proprie considerazioni via mail ethiektraining@ggdmn.nl entro lo scorso 15 marzo 2013.

\section{CONCLUSIONI}

I metodi di tipizzazione finalizzati alla definizione di un possibile outbreak devono rispondere a imprescindibili requisiti di:

- facilità di esecuzione

- rapidità

- riproducibilità intra laboratorio e inter laboratori

- costi contenuti

Quest'ultimo forse costituisce l'aspetto di minor peso specifico, se si considera che un difetto nell'accertamento epidemiologico di un outbreak soprattutto se sostenuto da germi multiresistenti comporta inevitabilmente un difetto nella messa in opera delle opportune misure di prevenzione e contenimento con un'inevitabile ricaduta sui costi diretti ed indiretti della gestione dell'outbreak in particolare e dell'ospedale in generale. Numerosi infatti sono gli elementi che, conseguentemente ad una cattiva gestione di un evento epidemico, pos- 
Tabella I. Metodi di tipizzazione a confronto

\begin{tabular}{|c|c|c|c|c|c|c|c|c|c|}
\hline & PFGE & RAPD & AFLP & RepPCR & MLVA & Rybotying & MLST & WGS & MALDI TOF \\
\hline Rapidità & 1 & 2 & 2 & 2 & 1 & 1 & 1 & 0 & 2 \\
\hline Semplicità & 2 & 2 & 1 & 2 & 2 & 2 & 1 & 0 & 2 \\
\hline Riproducibilità & 1 & 0 & 2 & 2 & 2 & 1 & 2 & 2 & 1 \\
\hline Potere discriminatorio & 0 & 0 & 1 & 1 & 1 & 0 & 2 & 2 & $1(?)$ \\
\hline Comparabilità & 2 & 0 & 0 & 2 & 2 & 1 & 2 & 2 & 1 \\
\hline Costi & $\begin{array}{l}1 \\
(20-30 €)\end{array}$ & $\begin{array}{l}2 \\
<10 €\end{array}$ & $\begin{array}{l}2 \\
12 €\end{array}$ & $\begin{array}{l}1 \\
20 €\end{array}$ & $\begin{array}{l}0 \\
30-100 €\end{array}$ & $\begin{array}{l}2 \\
<10 €\end{array}$ & $\begin{array}{l}0 \\
50 €\end{array}$ & $\begin{array}{l}0 \\
100 €\end{array}$ & $\begin{array}{l}2 \\
<2 €\end{array}$ \\
\hline Totale punteggio & 7 & 6 & 8 & 10 & 8 & 7 & 8 & 7 & 9 \\
\hline
\end{tabular}

Legenda: Punteggio assegnabile da 0 a 2; Rapidità = calcolato il tempo in ore, <24h (2); 24-48h (I); >48h (0);Semplicità $=$ numero di passaggi $\frac{c}{\lambda} 5=2 ; 5-10=1 ;>10=0 ;$ Riproducibilità $=$ se esistono o meno passaggi cruciali legati alla scelta operatore/condizioni fisico-chimiche dell'analisi; Potere discriminatorio $=$ da letteratura scarso $=0$; buono $=\mathrm{I}$, ottimo $=2$; Comparabilità $=$ possibilità di confronto via web scarsa $=0$; buona $=\mathrm{I}$, ottima $=2$; Costi $=$ $<20=2 ; 20-30=1 ;>30=0 .(4,6,9,10,13,16-22,24,25,27,29-34,35,37)$.

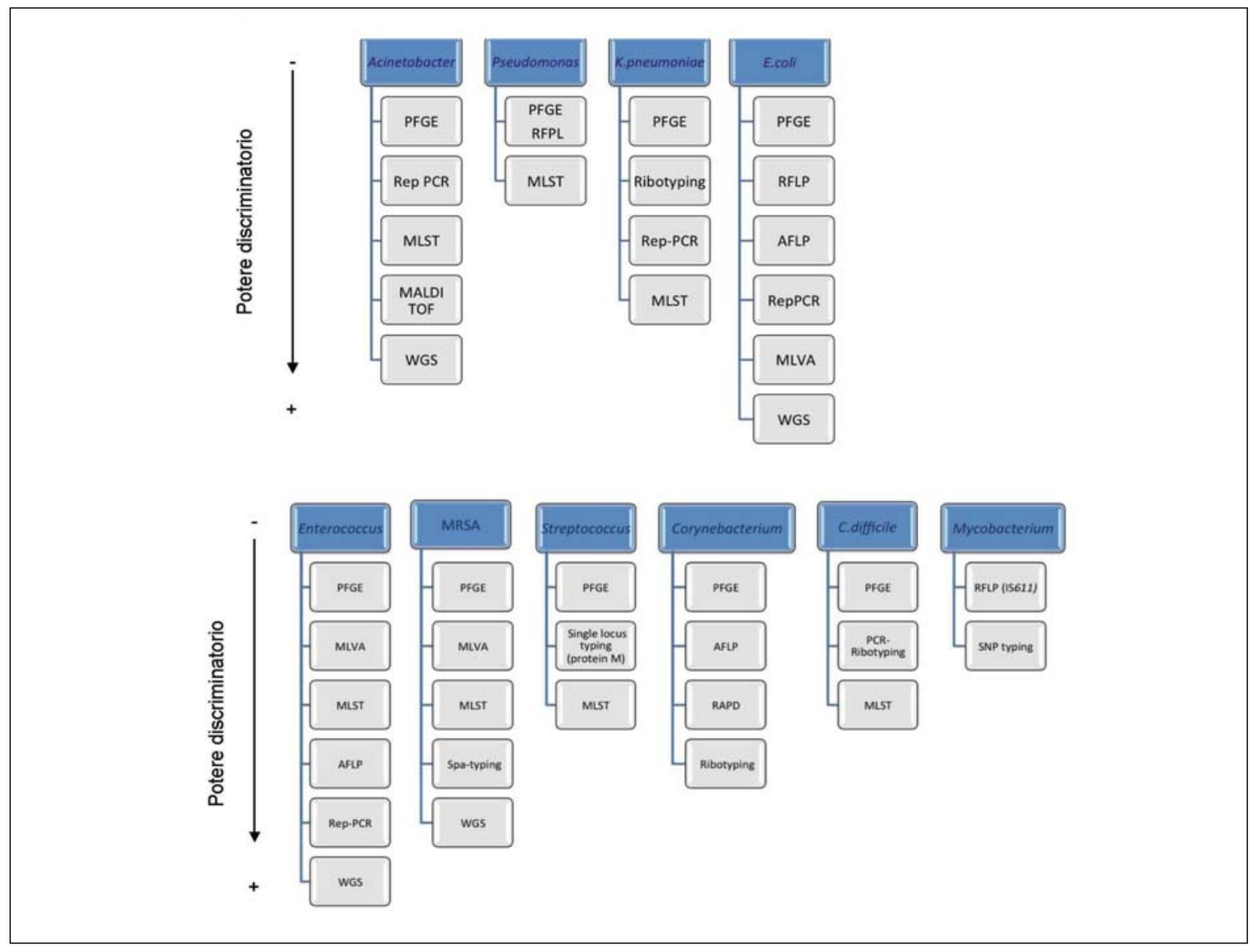

Figura I. Metodi di tipizzazione, ordinati in base al potere discriminatorio (crescente), per i principali gruppi di microrganismi

sono determinare un consumo di risorse economiche altrimenti evitabile maggiore utilizzo di antibiotici e altri presidi diagnostico-terapeutici, necessità di isolare pazienti e applicare speciali misure di prevenzione, prolungamento dei giorni di degenza e unitamente a questo deve essere considerato, oltre all'esito spesso sfavorevole delle infezioni sostenute da germi multi resistenti, l'impatto negativo sull'epidemiologia locale ad opera della pressione selettiva degli antibiotici utilizzati. Pregi e difetti dei vari metodi di tipizzazione vanno considerati, per il loro utilizzo, in relazione alla propria realtà di laboratorio e al proprio flusso di lavoro. Anche se alcuni aspetti fondamentali differenziano le diverse metodiche nel loro potere discriminante intra-isolato non esiste, in linea di principio, il sistema ideale e applicabile in maniera estensiva a tutti i microrganismi potenzialmente causa di outbreak. Occorre fare riferimento alla letteratura, in continuo aggiornamento, tenendo però presente che un sistema di tipizzazione per essere utile deve anzitutto poter discriminare fra 
loro con chiarezza isolati della stessa specie.

Anche in considerazione della riduzione delle risorse economiche disponibili occorre precisare che non tutti i laboratori possono impegnarsi nel fingerprinting ma, senza trascurare i concetti base della tipizzazione fenotipica, dovranno rivolgersi a centri di riferimento per la tipizzazione genomica dei propri isolati.

\section{Come conservare i ceppi}

Infine, un paragrafo destinato alla conservazione degli isolati dei sospetti eventi epidemici. L'esperienza di ogni microbiologo in questo caso è utile, ma si può semplicemente fare riferimento alle procedure raccomandate dall'American Type Culture Collection ATCC (38). Si tratta di preparare in una vial da crioconservazione una sospensione molto densa superiore al punto 2 della standard Mac Farland in brodo, quale: BHI addizionato del $20 \%$ di glicerolo da preferire al DMSO, spesso non gradito dai germi difficili e di conservare la sospensione a $-70^{\circ} \mathrm{C}$. In alternativa, per chi disponesse di un liofilizzatore, è indicata anche la liofilizzazione. Utile, per brevi periodi nell'ordine di 1 anno conservare in crio vials con $0.5 \mathrm{ml} \mathrm{di}$ sangue di montone intero defibrinato mantenendo le vials anche a $-20^{\circ} \mathrm{C}$. Più di recente è stata descritta una procedura che seziona e crio-conserva direttamente l'agar cioccolato su cui è stata effettuata una coltura del ceppo in esame, crioconservando i frammenti di agar a $-70^{\circ} \mathrm{C}$ (36).

\section{REFERENCES}

1. Beck-Saguè C, Jarvis WJ, Martone WJ. Outbreak investigation. Infect Control Hosp Epidemiol 1997; 18: 138-45.

2. Bouchet V, Huot H, Goldstein R. Molecular genetics of ribotyping. Clin Microbiol Rev 2008; 21: 262.

3. Cartwright CP. Polymerase chain reaction ribotyping: a "universal" approach? J Infect Dis 1995; 72: 1638-9.

4. Enright MC, Spratt BG. Multilocus sequence typing. Trends In Microb 1999; 7: 482-7.

5. Fontana C, Favaro M, Pistoia ES, et al. The combined use of VIGI@ct bioMérieux and fluorescent amplified length fragment polymorphisms in the investigation of potential outbreaks. J Hosp Infect 2007; 66: 262-8.

6. Fontana C, Favaro M, Minelli S, et al. Acinetobacter baumannii in intensive care unit: a novel system to study clonal relationship among the isolates. $B M C$ Infect Dis 2008; 8: 79.

7. Friedman C, Newson W, Arbor A. Basic concept of infection control. available at http://www.theific.org/basic_concepts/english_index.htm

8. Giannino V, Santagati M, Guardo G, Cascone C, Rappazzo G, Stefani S. Conservation of the mosaic structure of the four internal transcribed spacers and localisation of the rrn operons on the Streptococcus pneumoniae genome. FEMS Microbiol Lett 2003; 223: 245-52.

9. Griffin PM, Price GR, Schooneveldt JM, et al. Use of matrix-assisted laser desorption ionization-time of flight mass spectrometry to identify vancomycinresistant enterococci and investigate the epidemiology of an outbreak. Clin Microbiol 2012; 50: 2918-31.

10. Grimont F, Grimont PA. Ribosomal ribonucleic acid gene restriction patterns as potential taxonomic tools. Ann Inst Pasteur Microbiol 1986; 137: 165-75.

11. Gurtler V, Barrie HD. Typing of Staphylococcus aureus strains by PCR-amplification of variablelength $16 \mathrm{~S}-23 \mathrm{~S}$ rDNA spacer regions: characterization of spacer sequences. Microbiology 1995; 141: 1255-65.

12. Gurtler V, Stanisich VA. New approaches to typing and identification of bacteria using the 16S-23S rDNA spacer region. Microbiology 1996; 142: 3-16.

13. Healy M, Houng J, Bittner T, Lising M, Frye S, Raza S, Schrock P, Manry J, Renwick A, Nieto R, Woods C, Versalovic J, Lupski R. Microbial DNA typing by automated repetitive-sequenze-based PCR. J Clin Microbiol 2005; 43: 199-207.

14. Jayarao B.M, Dore JJ, Baumbach Jr GA, Matthews KR, Oliver SP. Differentiation of Streptococcus uberis from Streptococcus parauberis by polymerase chain reaction and restriction fragment length polymorphism analysis of $16 \mathrm{~S}$ ribosomal DNA. J Clin Microbiol 1991; 29: 2774-8.

15. Josten M, Reif M, Szekat C, et al. Analysis of the Matrix-Assisted Laser Desorption Ionization-Time of Flight Mass Spectrum of Staphylococcus aureus Identifies Mutations That Allow Differentiation of the Main Clonal Lineages. J Clin Microbiol 2013; 51: 1809-17.

16. http://www.keygene.com

17. Kolbert CP, Persing DH. Ribosomal DNA sequencing as a tool for identification of bacterial pathogens. Curr Opin Microbiol 1999; 2: 299-305.

18. Kostman JR, Edlind TD, LiPuma JJ, Stull TL. Molecular epidemiology of Pseudomonas cepacia determined by polymerase chain reaction ribotyping. $J$ Clin Microbiol 1992; 30: 2084-7.

19. Lagatolla C, Dolzani L, Tonin E, et al. PCR ribotyping for characterizing Salmonella isolates of different serotypes. J Clin Microbiol 1996; 34: 2440-3.

20. Loman NJ, Misra RV, Dallman TJ, et al. Performance comparison of benchtop high-throughput sequencing platforms. Nature biotechnology 2012; 30: 434-9.

21. Lupski JR, Weinstock GM. Short, interspersed repetitive DNA sequences in prokaryotic genomes. $J$ Bacteriol 1992; 174: 4525-9.

22. Maidak BL, Olsen GJ, Larsen N, Overbeek R, McCaughey MJ, Woese CR. The RDP Ribosomal Database Project. Nucleic Acids Res 1997; 25: 109-11.

23. Marsou R, Bes M, Boudouma M, et al. Distribution of Staphylococcus sciuri subspecies among human clinical specimens, and profile of antibiotic resistance. Res Microbiol 1999; 150: 531-41.

24. Maslow JN, Mulligan ME, Arbeit RD. Molecular epidemiology: application of contemporary techniques to the typing of microorganisms. Clin Infect Dis 1993; 17: 153-64.

25. Mencacci A, Monari C, Leli C, et al. Identification of nosocomial outbreaks of Acinetobacter baumannii using MALDI-TOF mass spectrometry. J Clin Microbiol 2012; doi:10.1128/JCM.01811-12.

26. Pourcel C, Minandri F, Hauck Y, et al. Identification of Variable-Number Tandem-Repeat VNTR Sequences in Acinetobacter baumannii and Interlaboratory Validation of an Optimized MultipleLocus VNTR Analysis Typing Scheme. J Clin Microbiol 2010; doi:10.1128/JCM.02003-10. 
27. Olsen GJ, Woese CR. Ribosomal RNA: a key to phylogeny. Faseb J 1993; 7: 113-23.

28. Raslan O. Outbreak Management Chapter 5 in IFIC Basic Concepts of Infection Control 2nd Edition Revised 2011 Editors Candace Friedman, William Newsom.

29. Sabat AJ, Budimir A, Nashev D, et al. Overview of molecular typing methods for outbreak detection and epidemiological surveillance. Eurosurveillance 2013, 18 issue 4.

30. Smith JJ, Scott-Craig JS, Leadbetter JR, Bush GL, Roberts DL, Fulbright DW. Characterization of random amplified polymorphic DNA RAPD products from Xanthomonas campestris and some comments on the use of RAPD products in phylogenetic analysis. Mol Phylogenet Evol 1994; 3: 135-45.

31. Stefani S, Chung DR, Jodi A, et al. Meticillin-resistant Staphylococcus aureus MRSA: global epidemiology and harmonisation of typing methods. International Journal of Antimicrobial Agents 2012; 39: 273-82.

32. Tyler KD, Wang G, Tyler SD, Johnson WM. Factors Affecting Reliability and Reproducibility of Amplification-Based DNA Fingerprinting of Representative Bacterial Pathogens. J Clin Microbiol 1997; 35: 339-46.
33. Vauterin L, Vauterin P. Integrated databasing and analysis. In: Molecular Identification, Systematics, and Population Structure of Prokaryotes ed. Erko Stackebrandt. Springer, 2006.

34. Versalovic J, Schneider M, de Bruijn FJ, Lupski JR. Genomic fingerprinting of bacteria using repetitive sequenced-based polymerase chain reaction. Meth Cell Mol Biol 1994; 5: 25-40.

35. Vos P, Hogers R, Bleeker M, et al. AFLP: a new technique for DNA fingerprinting. Nucleic Acids Res 1995; 23: 4407-4.

36. Winters RD, Winn WC, Simple Jr A. Effective Method for Bacterial Culture Storage: A Brief Technical Report. Journal of Bacteriology and Virology.2010; 40 2: 99-101.

37. Xiao D, You Y, Bi Z, et al. MALDI-TOF mass spectrometry-based identification of group A Streptococcus isolated from areas of the 2011 scarlet fever outbreak in china. Infect Genet Evol 2013; 14: 320-6.

38. http://www.atcc.org/ /media/PDFs/Culture\%20Guides/A TCC_Bacterial_Culture_Guide.ashx.

39. Tura $\overline{-}$, Moro $\overline{M L}$, Caminati A, et al. Epidemie di Infezioni Correlate all'assistenza sanitaria. Dossier 123 Agenzia Sanitaria Regionale Emilia-Romagna 2006. 